\title{
Coherent Electron Scattering Captured by an Attosecond Quantum Stroboscope
}

\author{
J. Mauritsson, ${ }^{1,2}$ P. Johnsson, ${ }^{1,3}$ E. Mansten, ${ }^{1}$ M. Swoboda, ${ }^{1}$ T. Ruchon, ${ }^{1}$ A. L'Huillier, ${ }^{1}$ and K. J. Schafer ${ }^{2}$ \\ ${ }^{1}$ Department of Physics, Lund Institute of Technology, P. O. Box 118, SE-221 00 Lund, Sweden \\ ${ }^{2}$ Department of Physics and Astronomy, Louisiana State University, Baton Rouge, Louisiana 70803-4001, USA \\ ${ }^{3}$ FOM-Institute AMOLF, Kruislaan 407, 1098 SJ Amsterdam, The Netherlands
}

(Received 15 November 2007; published 21 February 2008)

\begin{abstract}
We demonstrate a quantum stroboscope based on a sequence of identical attosecond pulses that are used to release electrons into a strong infrared (IR) laser field exactly once per laser cycle. The resulting electron momentum distributions are recorded as a function of time delay between the IR laser and the attosecond pulse train using a velocity map imaging spectrometer. Because our train of attosecond pulses creates a train of identical electron wave packets, a single ionization event can be studied stroboscopically. This technique has enabled us to image the coherent electron scattering that takes place when the IR field is sufficiently strong to reverse the initial direction of the electron motion causing it to rescatter from its parent ion.
\end{abstract}

DOI: 10.1103/PhysRevLett.100.073003

PACS numbers: $32.80 . \mathrm{Rm}$

The basic properties of atoms, molecules, and solids are governed by electron dynamics which take place on extremely short time scales. To measure and control these dynamics therefore requires ultrafast sources of radiation combined with efficient detection techniques. The realization of extreme ultraviolet (XUV) attosecond ( 1 as = $10^{-18} \mathrm{~s}$ ) pulses $[1,2]$ has, for the first time, made direct measurements of electron dynamics possible [3-5]. Pioneering experiments utilizing the fast varying electric fields of femtosecond infrared (IR) laser pulses have demonstrated that temporally localized electron wave packets (EWPs) can be used to study molecular structures and dynamics [6-8]. In these experiments, subfemtosecond EWPs are generated through tunnel ionization twice per optical cycle near the maxima in the laser's oscillating electric field. They are subsequently accelerated by the same laser field and may be driven back to their parent ion for further interaction. This basic sequence of events, which is the essence of strong field physics, is very versatile and leads to many different phenomena $[9,10]$. The only control knob in these experiments, however, is typically the laser intensity, which must be quite high in order to ensure a reasonable probability of tunneling through the Coulomb barrier.

Further control of the electron dynamics requires that the creation and acceleration of the EWPs are decoupled; this is not possible using tunneling ionization since the same laser field governs both events. Decoupling can be achieved by using XUV attosecond pulses to create temporally localized EWPs through single photon ionization at a well defined phase of a synchronized IR field which drives the dynamics from that point forward. These attosecond EWPs are distinctly different from their tunnel ionization counterparts. They are born at the center of the potential well with properties that are directly inherited from the XUV pulses, which can be tailored in time and frequency [11-15]. They can also have a nonzero velocity, and their subsequent dynamics can be controlled by choos- ing the phase and amplitude of a synchronized IR field appropriately. In particular, the laser field needed to drive these EWPs back to the potential is usually weaker than the laser field needed to form tunnel EWPs, leading to much less distortion of the properties to be studied.

Here, we demonstrate an attosecond quantum stroboscope capable of capturing electron motion on a subfemtosecond time scale. This technique is based on a sequence of identical attosecond pulses [11] which are synchronized with an IR laser field. The pulse to pulse separation in the train is tailored to exactly match an optical cycle of the laser field, and the electron momentum distributions are detected with a velocity map imaging spectrometer (VMIS) [16,17]. This technique has enabled us to image the coherent scattering of electrons that are driven back to the ion by the laser field following their ionization. We envision that coherent electron scattering from atoms, molecules, and surfaces captured by the attosecond quantum stroboscope will complement more traditional scattering techniques $[18,19]$ since it provides high temporal as well as spatial resolution [20].

To understand the principle of the quantum stroboscope and its application to coherent electron scattering, we use a simple classical model, where the atomic potential is omitted, to describe the motion of an electron that is released in a laser field [21]. If the electron is created at rest at time $t_{0}$ in a linearly polarized laser field with $\mathbf{E}(t)=\hat{\mathbf{y}} E_{0} \sin (\omega t)$, it will at a later time $t$ have a momentum

$$
\mathbf{p}\left(t, t_{0}\right)=\frac{e E_{0} \hat{\mathbf{y}}}{\omega}\left[\cos \left(\omega t_{0}\right)-\cos (\omega t)\right],
$$

where $e$ is the electron charge. Equation (1) is useful to describe tunneling wave packets, which are born at rest, but not sufficient to describe EWPs that are created via photoabsorption from attosecond pulses since they will have an initial momentum $\mathbf{p}_{0}=\sqrt{2 m W} \hat{\mathbf{u}}$, where $W=$ $E_{\mathrm{XUV}}-I_{p}$ is the kinetic energy, $m$ the mass of the electron, $I_{p}$ the ionization energy, and $\hat{\mathbf{u}}$ is the initial direction of the 
outgoing electron. Introducing the dimensionless parameter $\tilde{\gamma}=\sqrt{W / 2 U_{p}}$ [22], where the quiver energy $U_{p}=$ $e^{2} E_{0}^{2} / 4 m \omega^{2}$, Eq. (1) is generalized to

$$
\mathbf{p}\left(t, t_{0}\right)=\frac{e E_{0}}{\omega}\left\{\left[\cos \left(\omega t_{0}\right)-\cos (\omega t)\right] \hat{\mathbf{y}}+\tilde{\gamma} \hat{\mathbf{u}}\right\} .
$$

Equation (2) describes the wiggling motion of the electron while it is in the laser field. We detect the final (drift) momentum:

$$
\mathbf{p}_{\mathbf{f}}\left(t_{0}\right)=\frac{e E_{0}}{\omega}\left[\cos \left(\omega t_{0}\right) \hat{\mathbf{y}}+\tilde{\gamma} \hat{\mathbf{u}}\right] .
$$

In order to observe coherent scattering, the electron, once released, must pass the ion core at least once. From Eq. (3), the final momentum will be zero or opposite to the initial momentum, for certain time delays, if $\hat{\mathbf{u}} \| \hat{\mathbf{y}}$ and $\tilde{\gamma}$ is smaller than or equal to 1 . When $\tilde{\gamma}=1$, the momentum transferred by the field to the electrons is such that only electrons that are born exactly at times when $\mathbf{E}(t)=0$ will return to the ion since the net transfer of momentum from the laser field (proportional to the vector potential of the field) is maximized for these times. For smaller $\tilde{\gamma}$-values, the momentum transfer is larger, which means that the initial direction of the electron can be reversed for a range of initial times. $\tilde{\gamma}=1$ therefore marks the boundary, in this simple model, between "strong" and "weak" field continuum dynamics for the electron. For a $800 \mathrm{~nm}$ laser wavelength, $\tilde{\gamma}=1$ can be obtained with $I=1 \times$ $10^{13} \mathrm{~W} / \mathrm{cm}^{2}$ and $W=1.2 \mathrm{eV}$. This intensity is an order of magnitude smaller than that needed for tunnel ionization.

The principle of the quantum stroboscope technique used to capture the electron motion is illustrated in Figs. 1(a) and 1(b). A sequence of identical attosecond pulses are used to release electrons into an IR laser field exactly once per laser cycle. The EWPs disperse as they fly towards the detector where their momentum distribution is recorded. Consecutive EWPs will therefore overlap and interfere. Since the impulse imparted to the electron by the IR field depends on when the ionization occurs [23-25], each phase of the oscillating laser field yields a unique final momentum distribution. When the attosecond pulse periodicity matches the IR optical cycle, the created EWPs are affected identically by the IR field, with the result that the dynamics of individual events can be studied stroboscopically. In contrast, if ionization occurs over the whole IR cycle, or even at as few as 2 times during the cycle, the resulting momentum distribution will be smeared out and show interference fringes that depend on the different ionization times [26]. The quantum stroboscope shares two important features with a conventional stroboscope: First, we can freeze the periodically varying momentum distribution at a single phase of the IR field, and capture the entire time-dependent distribution by varying the XUV-IR delay. Second, by repeating the process periodically, the measured signal is stronger than what we would measure
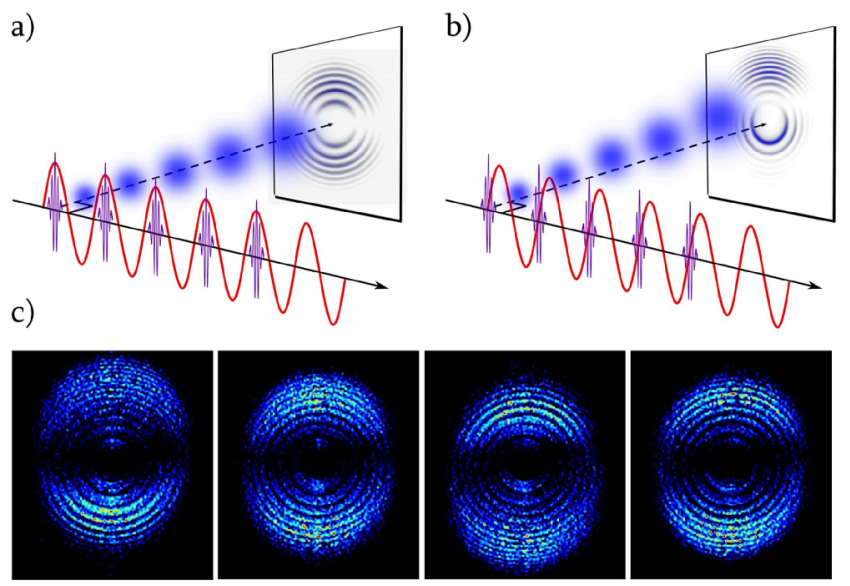

FIG. 1 (color online). (a), (b) An APT is used to ionize the target atoms once per cycle of an IR laser field. When the EWPs are created at the maxima of the IR electric field (a), the net transfer of momentum is zero, and the resulting momentum distribution is symmetric relative to the plane perpendicular to the laser polarization. When the EWPs instead are created at the zero-crossings of the IR electric field (b), the momentum distribution is shifted by the field along the direction of the laser polarization. (c) Experimental results obtained in Ar at four different XUV-IR delays are shown. From left to right, the images correspond to the XUV-IR delays $t_{0}=0, \pi / 2 \omega, \pi / \omega$, $3 \pi / 2 \omega$ for an IR intensity of $5 \times 10^{12} \mathrm{~W} / \mathrm{cm}^{2}$.

with a single pulse. In the quantum stroboscope, the signal is further enhanced due to the coherence of the process, in that a train of $N$ pulses yields fringes that are $N^{2}$ times brighter than the signal from an isolated pulse. The quantum stroboscope technique thus provides a complementary approach to the use of isolated attosecond pulses, in particular, suitable for experiments where a high signal to noise ratio is required.

In a first experimental demonstration of this technique, we use pulses with a 300 as duration and a central energy of $24 \mathrm{eV}$ to ionize argon in the presence of an IR laser field with an intensity of $5 \times 10^{12} \mathrm{~W} / \mathrm{cm}^{2}$. The attosecond pulse train (APT) was generated from a two-color laser field consisting of the IR field and its second harmonic to ensure that the XUV pulses were separated by one full optical cycle [11]. Four stroboscopic images taken at different XUV-IR delays $\left(t_{0}\right)$ are presented in Fig. 1(c) (a complete movie spanning one full optical cycle is available in Ref. [27]). The clear up or down asymmetry in the momentum distributions confirms that each image corresponds to ionization at one particular phase of the IR field so that the momentum distribution is shifted up or down in the direction of polarization of the IR field. These results illustrate that the two essential aspects of stroboscopic imaging mentioned above are fulfilled: the electron momentum distribution is "frozen" at a given phase of the IR field, and the signal strength is enhanced by the repetition of the process. The interference fringes, which are evenly 
spaced one IR photon apart in energy, show a decreasing spacing when plotted as a function of momentum as in Fig. 1. The position of these fringes does not shift as a function of XUV-IR delay, which shows that we are imaging a train of identical EWPs spaced one IR cycle apart. In addition, the fringe positions depend only on the IR intensity. The quantum stroboscope is therefore self-calibrating since the only unknown parameter, the IR intensity, can be read directly from the interference pattern. In this experiment $\tilde{\gamma}=3.7$; i.e., the field strength was not sufficient with respect to the initial energy of the electrons to reverse their initial velocity and coherent electron scattering is therefore not expected to be observed.

To understand what we can expect from experiments that guide ionized electrons to rescatter off the ion core, we have performed a series of calculations in helium by numerically integrating the time-dependent Schrödinger equation (TDSE) [28,29]. In Fig. 2, we show the influence of $\tilde{\gamma}$ on momentum distributions obtained at the XUV-IR delay leading to the maximum momentum transfer. The white circles in each panel indicate the range of momenta predicted by Eq. (3) if there is no rescattering from the ion [23]. When the intensity is increased, the downward momentum region extends outside the white circle, which indicates that electrons whose initial direction has been reversed have scattered off the atomic potential and that they have gained extra momentum in the same direction as their initial direction [30,31]. Another interference pattern is readily seen, with maximum along the polarization axis and minima at approximately $30^{\circ}$ from the polarization axis (region II). This interference occurs between the "direct" outgoing EWP and the "rescattered" one. This interference is similar to the holographic imaging proposed by Spanner and co-workers using above threshold ionization [32]. When $\tilde{\gamma}=0.86$, a larger portion of the EWP scatters of the potential, and the scattered electrons can be seen as jets along the polarization direction. A more de- tailed analysis of these effects is outside the scope of this Letter and will be discussed in a forthcoming theoretical paper.

To experimentally image coherent electron scattering with the quantum stroboscope, we ionize helium atoms with $E_{\mathrm{XUV}}=25.8 \mathrm{eV}(W=1.2 \mathrm{eV})$ and an IR intensity $I=1.2 \times 10^{13} \mathrm{~W} / \mathrm{cm}^{2}$. In this case, $\tilde{\gamma} \approx 0.9$; i.e., the laser field is sufficiently strong to drive the electrons back to the ion core for some XUV-IR delays. Compared to argon, the helium momentum distributions are expected to be more peaked along the laser polarization direction, since the excited EWP is entirely in an $m=0$ state, whereas for argon, there is a mixture of $m=0$ and $m=$ 1 states, and the latter has no amplitude along the polarization axis. This makes helium a better candidate to observe electron scattering since the electrons along the polarization direction have the highest probability to scatter off the potential. Four experimental momentum distributions recorded at different XUV-IR delays are presented in Fig. 3. When the XUV-IR timing is set to maximize the momentum transfer from the IR field in the upwards (panel 1) or downwards (panel 3) directions, we see a clear signature of rescattering, manifested by a significant increase of low-energy electrons in the direction opposite to the momentum transfer from the IR field [33]. The experimental results are compared with theoretical calculations in the first panel of Fig. 3, and the agreement is excellent with all the substructures well reproduced. We believe that this is the first evidence for coherent electron scattering of attosecond EWPs created by single photon ionization.

In this Letter, we have demonstrated an attosecond quantum stroboscope capable of imaging the electron momentum distribution resulting from a single ionization event. We have also used it to guide ionized electrons back to their parent ions and to image the coherent electron scattering. The basic technique we have demonstrated is very versatile and may be altered in a number of potentially
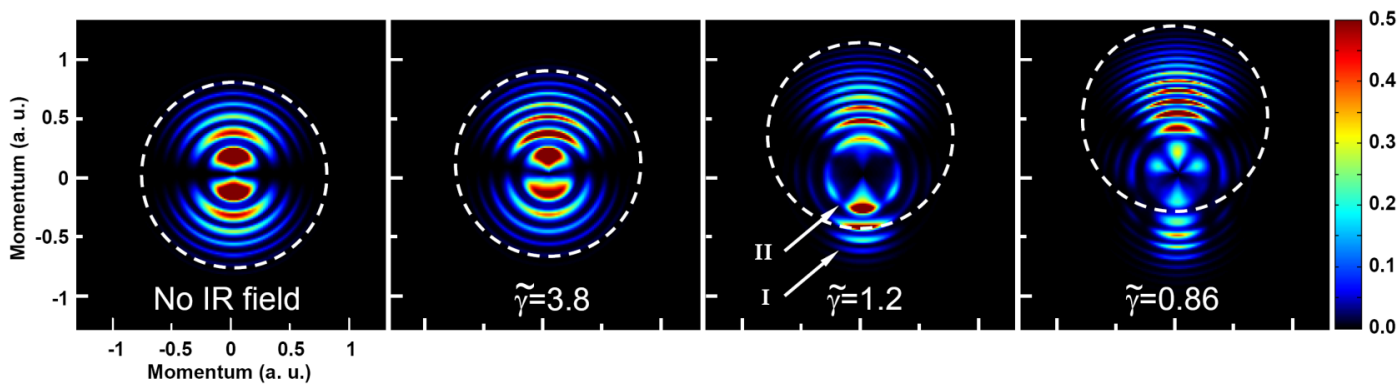

FIG. 2 (color online). Theoretical results obtained by integrating the TDSE are shown (from left to right) for IR intensities equal to zero, $1 \times 10^{12} \mathrm{~W} / \mathrm{cm}^{2}, 1 \times 10^{13} \mathrm{~W} / \mathrm{cm}^{2}$, and $2 \times 10^{13} \mathrm{~W} / \mathrm{cm}^{2}$ for the XUV-IR delay which corresponds to the maximum momentum transfer. The white circles are positioned at the highest energy electrons in the field free case (panel 1) and shifted by the amount of momentum added by the IR field in the other panels. If no post-ionization interaction between the electron and the atom occurs, the momentum distributions would remain within the circles. Two features are highlighted in panel three, which is calculated at an intermediate intensity where $\tilde{\gamma}=1.2$, by white arrows: (I) electrons that have scattered off the core appearing outside the white circle in the downward direction and (II) interference minima in the momentum distribution. The interference minima occur in the region where the rescattered and direct electrons overlap. 

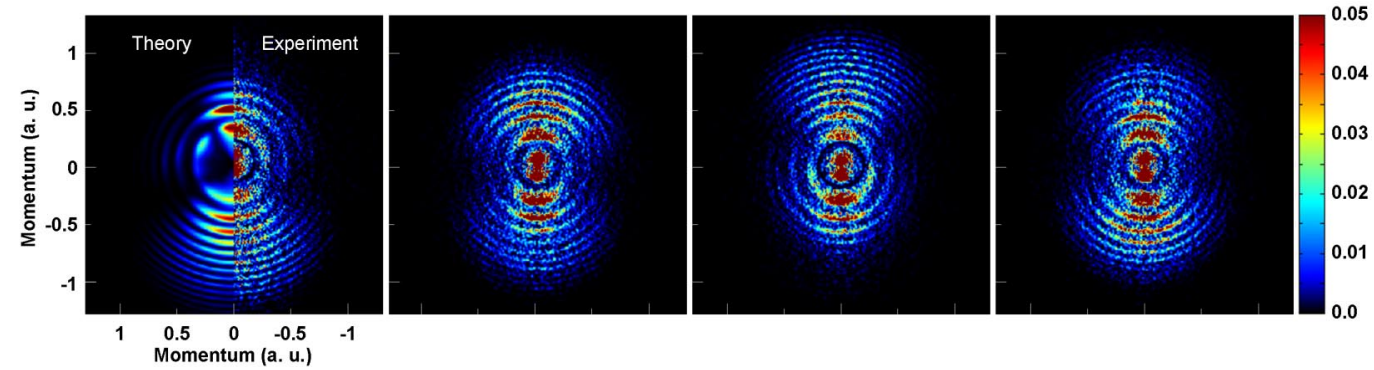

FIG. 3 (color online). Experimental results obtained in helium at an intensity of $1.2 \times 10^{13} \mathrm{~W} / \mathrm{cm}^{2}$ are shown. The results are distinctively different from those taken in argon (Fig. 1). With this higher intensity, more momentum is transferred to the electrons, and in combination with the lower initial energy, some electrons return to the atomic potential for further interaction. In the first panel, we compare the experimental results (right) with theoretical calculations (left) obtained for the same conditions. The excellent agreement is the strongest evidence for coherent scattering effects in the experiment. All the substructures are well reproduced except for the highly saturated innermost peak in the experiment, which most likely is due to above threshold ionization of residual water in the experimental chamber.

useful ways. For example, the guiding field could be a replica of the two-color driving field we used to make the attosecond pulses. This would provide additional control over the return time and energy of the electrons. Using a longer wavelength driving laser [34] would lengthen the time between attosecond pulses, allowing more time for internal dynamics initiated by the launch of the EWP to develop before being probed by the returning electron. We envision that controlled, coherent scattering such as we have demonstrated will enable time resolved measurements with very high spatial resolution in atoms and molecules or at surfaces.

This research was supported by the Marie Curie IntraEuropean Fellowship (Attotech), the Marie Curie Research Training Networks (XTRA), the Marie Curie Early Stage Training Site (MAXLAS), the Integrated Initiative of Infrastructure LASERLAB-EUROPE within the 6th European Community Framework Programme, the Knut and Alice Wallenberg Foundation, the Crafoord Foundation, the Swedish Research Council, and the National Science Foundation (Grant No. PHY-0701372).

[1] P. M. Paul et al., Science 292, 1689 (2001).

[2] M. Hentschel et al., Nature (London) 414, 509 (2001).

[3] M. Drescher et al., Nature (London) 419, 803 (2002).

[4] R. Kienberger et al., Nature (London) 427, 817 (2004).

[5] E. Goulielmakis et al., Science 305, 1267 (2004).

[6] H. Niikura et al., Nature (London) 417, 917 (2002).

[7] J. Itatani et al., Nature (London) 432, 867 (2004).

[8] T. Kanai, S. Minemoto, and H. Sakai, Nature (London) 435, 470 (2005).

[9] K. J. Schafer et al., Phys. Rev. Lett. 70, 1599 (1993).

[10] P. B. Corkuum, Phys. Rev. Lett. 71, 1994 (1993).

[11] J. Mauritsson et al., Phys. Rev. Lett. 97, 013001 (2006).

[12] R. López-Martens et al., Phys. Rev. Lett. 94, 033001 (2005).
[13] E. Gustafsson et al., Opt. Lett. 32, 1353 (2007).

[14] G. Sansone et al., Science 314, 443 (2006).

[15] I. J. Sola et al., Nature Phys. 2, 319 (2006).

[16] M. J. J. Vrakking, Rev. Sci. Instrum. 72, 4084 (2001).

[17] A. T. J. B. Eppink and D. H. Parker, Rev. Sci. Instrum. 68, 3477 (1997).

[18] J. Miao, P. Charalambous, J. Kirz, and D. Sayre, Nature (London) 400, 342 (1999).

[19] H. N. Chapman et al., Nature Phys. 2, 839 (2006).

[20] H. Niikura and P. B. Corkum, Adv. At. Mol. Opt. Phys. 54, 511 (2007).

[21] T. F. Gallagher, Phys. Rev. Lett. 61, 2304 (1988).

[22] G. Lagmago Kamta and A. F. Starace, Phys. Rev. A 68, 043413 (2003).

[23] J. Itatani et al., Phys. Rev. Lett. 88, 173903 (2002).

[24] R. Kienberger et al., Science 297, 1144 (2002).

[25] P. Johnsson et al., Phys. Rev. Lett. 95, 013001 (2005).

[26] K. C. Kulander, K. J. Schafer, and J. L. Krause, in Atoms in Intense Laser Fields (Academic Press, San Diego, 1992).

[27] See EPAPS Document No. E-PRLTAO-100-004808 for a movie spanning one full optical cycle. For more information on EPAPS, see http://www.aip.org/pubservs/epaps .html.

[28] J. L. Krause, K. J. Schafer, and K. C. Kulander, Phys. Rev. Lett. 68, 3535 (1992).

[29] T. Remetter et al., Nature Phys. 2, 323 (2006).

[30] O. Smirnova, S. Patchkovskii, and M. Spanner, Phys. Rev. Lett. 98, 123001 (2007).

[31] A. K. Kazansky and N. M. Kabachnik, J. Phys. B 40, 2163 (2007).

[32] M. Spanner, O. Smirnova, P. B. Corkum, and M. Y. Ivanov, J. Phys. B 37, L243 (2004).

[33] We note that there is an up/down asymmetry even for the XUV-IR delays where no net momentum is transferred to the electrons. We attribute this asymmetry partially to a small frequency modulation on the attosecond pulses and partially to the distortion of the atomic potential.

[34] P. Agostini and L. F. DiMauro, Rep. Prog. Phys. 67, 813 (2004). 